\title{
An Experimental Study of Transvenous Defibrillation Using a Coronary Sinus Catheter
}

\author{
ALAN H. KADISH, M.D., KEITH CHILDS, B.S., and JOSEPH LEVINE, M.D.
}

From the Division of Cardiology, Department of Internal Medicine, University of Michigan Medical Center, Ann Arbor, Michigan, and the Division of Cardiology, St. Francis Hospital and Cardiac Center, Roslyn, New York

KADISH, A.H., ET AL.: An Experimental Study of Transvenous Defibrillation Using a Coronary Sinus Catheter. The efficacy of a transvenous defibrillating system, utilizing bipolar right ventricular and coronary sinus catheters was evaluated in 14 normal mongrel dogs. Two groups of seven animals each were studied. During all shocks, the right ventricular apex electrode served as the anode. In both groups, defibrillation was performed using the proximal pole of the right ventricular catheter (superior vena cava), as the cathode served as a control (configuration A). In group 1, a coronary sinus cathode (configuration B) was compared to control. The mean energy at which $50 \%$ or more of the shocks were successful was similar for configuration $B(20.7 \pm 7.9$ joules) and for configuration $A(18.8 \pm 9.4$ joules $)$. In group 2 , the superior vena cava and coronary sinus electrodes served as a common cathode (configuration C). Mean defibrillation energy at which $50 \%$ or more of the shocks was successful was $21.4 \pm 9.0$ joules for configuration $C$ and $27.1 \pm$ 9.5 joules for configuration $A(P<0.01)$. Leading edge voltage was similar for all three configurations, but shock duration was longer for configuration $A(11.3 \pm 2.8 \mathrm{msec})$ than configuration $B(6.6 \pm 1.8 \mathrm{msec})$ or $C(6.1 \pm 1.5 ; P<0.05)$. Nonsustained ventricular tachycardia and transient heart block were common, but no damage to the coronary sinus was noted despite the delivery of up to 38 shocks. Conclusions: (1) With the catheter system used, coronary sinus to right ventricular apex defibrillation system offered no advantages over a superior vena cava to right ventricular apex system; (2) A three-electrode system with the high right atrium and coronary sinus serving as the common cathode reduced defibrillation thresholds significantly without any severe short-term adverse consequences; and (3) Improvements in catheter design may make a coronary sinus catheter part of a feasible transvenous defibrillating system. (J Electrophysiol $3: 253-260,1989$ )

defibrillation, AICD, catheter defibrillation, coronary sinus, ventricular fibrillation

The use of the automatic implantable cardioverter defibrillator (AICD) has resulted in a decrease in mortality in patients suffering lifethreatening cardiac arrhythmias. ${ }^{1-5}$ However, significant morbidity is caused by the current implantation procedure involving a thoracotomy, subxyphoid or intercostal incision, and implantation of one or two epicardial patch electrodes. ${ }^{6-7}$ Recently, a nonthoracotomy device

Supported in part by Grant 40667-1 from the National Institute of Health, Bethesda, Maryland.

Address for correspondence: Alan H. Kadish, M.D., University of Michigan Hospital, 1500 E. Medical Center Drive, Cardiology, B1-F245, Ann Arbor, MI 48109-0022. involving a subcutaneous patch placed in the left chest has undergone experimental and preliminary clinical testing. Defibrillation thresholds have been somewhat variable using this configuration. ${ }^{8}$

Transvenous defibrillation was investigated early in the development of the AICD using a bipolar electrode placed in the right ventricular apex with the proximal pole being located in the high right atrium. A recent experimental study has investigated defibrillation thresholds using this configuration and found a mean threshold of 26 joules. ${ }^{9}$ Since successful defibrillation appears to be related to the volume of the left ventricle that is subjected to 
an adequate current density during the shock, ${ }^{10}$ we hypothesize that placing a catheter in the coronary sinus in addition to the right ventricular apex might interpose a larger portion of myocardium between the defibrillating electrodes and thus lead to a lowering of the transvenous defibrillation threshold.

\section{Methods}

\section{Surgical Procedure}

Adult mongrel dogs with a mean weight of $21.6 \mathrm{~kg}$ were anesthetized intravenously with sodium pentobarbital, $25 \mathrm{mg} / \mathrm{kg}$ and ventilated with a volume ventilator (Harvard Apparatus). The right femoral vein was cannulated for intravenous access, both jugular veins were isolated, and a carotid artery cannulated for blood pressure measurement. The chest was opened via a median sternotomy and pericardial cradle was created.

Two custom-made tripolar catheters (CPI, St. Paul, MN, USA) were used for defibrillation. The catheters had a small tip electrode and two large proximal defibrillating electrodes. The tip electrode was used (along with the distal large electrode) for the induction of fibrillation and was $6 \mathrm{~mm}$ in length and had a surface area of $9.0 \mathrm{~mm}^{2}$. The proximal defibrillating electrode was $73 \mathrm{~mm}$ in length, the distal electrode was $35 \mathrm{~mm}$ in length, and the interelectrode distance was $62 \mathrm{~mm}$. Catheter diameter was $3 \mathrm{~mm}$, distal electrode surface area was $277 \mathrm{~mm}^{2}$, and proximal electrode surface area was $578 \mathrm{~mm}^{2}$. One catheter was placed in the coronary sinus (CS) via the left jugular vein and the second in the right ventricular apex through the right jugular vein. The proximal pole of the right ventricular catheter was in the distal superior vena cava (SVC). Blood pressure was recorded using a fluid-filled catheter. Electrocardiograms and blood pressure were amplified and displayed on an Electronics for Medicine Oscilloscope (Electronics for Medicine, Pleasantville, NY, USA) and recorded on heat sensitive paper.

Ventricular fibrillation was induced through the right ventricular catheter using alternating current. An external cardioverter defibrillator (CPI) connected to the transvenous leads was used for defibrillation. If the transvenous shocks were unsuccessful, dogs were rescued with internal paddles connected to a standard defibrillator. Bipolar Teflon-coated silver wire pacing electrodes were threaded with a 21-gauge needle into the subepicardium. A Bloom DTU-110 Stimulator (Bloom Assoc., Norbeth PA, USA) was used to pace the heart at a cycle length of $500 \mathrm{msec}$ if temporary heart block lasting more than 3 seconds developed following fibrillation and defibrillation.

\section{Experimental Protocol}

Two groups of seven animals each were studied to examine the use of a coronary sinus catheter in a bipolar (group 1) or tripolar (group 2) configuration. In both groups, a right ventricular apex anode and a superior vena cathode (configuration A) both of which were on the same catheter served as the control. In each group, a test configuration was compared to configuration A. In group 1, defibrillation using the distal coronary sinus cathode and right ventricular apex anode (configuration B) was compared to control. In group 2, configuration C which consisted of the right ventricular apex as the anode and the superior vena cava (proximal right ventricular catheter) and distal coronary sinus as a common cathode was compared to control.

A truncated exponential waveform was delivered at a pulse energy varying between 5-40 joules. Defibrillating attempts began 12 seconds after the induction of fibrillation. At least 5 minutes elapsed between defibrillation attempts. In order to account for the variability in defibrillation success at a given energy, 11,12 a crude defibrillation threshold (DFT) was first identified and then multiple shocks were delivered around that energy to perform probability calculations. To determine the crude DFT, the first shock was delivered at a 40 -joules energy level. If defibrillation was successful, energy output was decreased in 5-joules steps to 10 joules and 2-joules steps below that. At least 5 minutes elapsed between defibrillation attempts. After the crude DFT was identified, 
up to five shocks were delivered using each configuration at three energy levels adjacent to the crude DFT to allow probability calculations. At each energy level, shocks using one of the configurations was available for comparison with the other configuration. Animals were included for analysis if at least three pairs of the shocks could be delivered at each of these three adjacent energy levels. Ventricular tachycardia was defined as that having a uniform beat to beat morphology and lasting more than 6 seconds following the delivery of a defibrillation shock.

Voltage waveforms were monitored during defibrillation shocks using a standard $10 x$ voltage probe (Tektronix, model P6122, Tektronix, Beaverton, OR, USA) with an input impedance of $11 \mathrm{Mohms}$. Voltage waveforms were displayed on an oscilloscope and photographed on Polaroid film (Polaroid, Boston, MA, USA).

\section{Data Analysis}

Data are expressed as mean \pm SD unless otherwise indicated. The successful energy mean was computed from the successful total energy at each configuration divided by the number of successful shocks. Defibrillation efficacies were compared by defining the energy at which more than $50 \%$ of shocks were successful in a given configuration $\left(\mathrm{E}_{50}\right)$. Variability in heart rate and blood pressure were analyzed using a coefficient of variation. The incidence of ventricular tachycardia and heart block were compared by Chi-square analysis with Yates continuity correction. Voltage measurements in the different configurations were compared using repeated measures analysis of variance using Scheffe's F-test.

\section{Results}

The animals remained physiologically stable through the multiple shock procedure. Mean systolic blood pressure was $116 \pm 17 \mathrm{mmHg}$, mean diastolic blood pressure was $91 \pm 21$ $\mathrm{mmHg}$, and mean pulse was $156 \pm 22$ beats/min. Groups A and B had similar pressure and pulse measurements during the pro- tocol. There was no significant change in blood pressure or pulse with time (Fig. 1).

\section{Defibrillation}

Mean weight was $19.6 \pm 6.6 \mathrm{~kg}$ in group 1 . A total of 196 shocks were analyzed in group 1 (mean 28 per animal per configuration). One hundred and ten (56\%) were successful. There was no significant difference in the number of successful shocks between the two configurations. Fifty-four shocks (55\%) were successful using configuration B (RV apex to distal coronary sinus) and 56 shocks using configuration A $(57 \% ; \mathrm{P}=\mathrm{NS})$. Mean defibrillation energy at which $50 \%$ or more of the shocks were

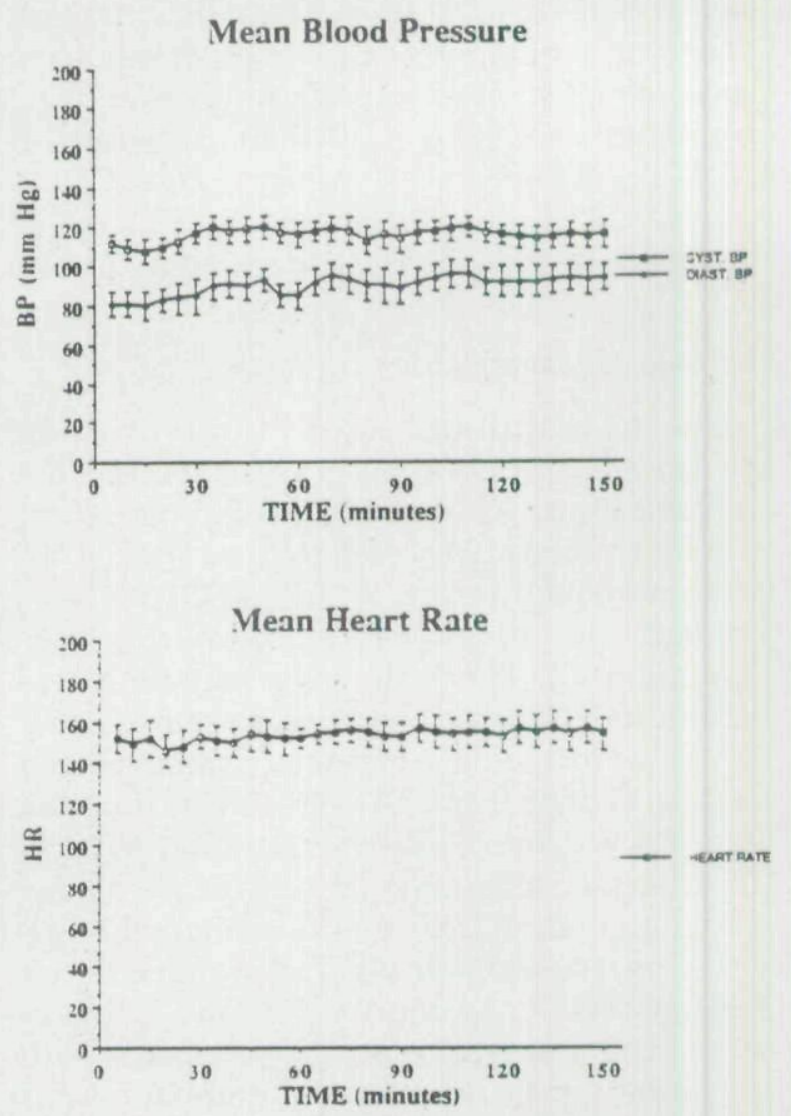

Figure 1. Mean blood pressure $(A)$ and pulse $(B)$ in beats per minute for all animals during the experimental procedure. Data are shown for the $3 \frac{1}{2}$ hours after the start of defibrillation attempts. There was no significant change in blood pressure or heart rate as the experiment progressed. 
successful $\left(\mathrm{E}_{50}\right)$ was $20.7 \pm 7.9$ joules for configuration $B$ and $18.8 \pm 9.4$ joules for configuration $A(P=N S)$. Values for $E_{50}$ in individual animals are shown in Figure 2. There was also no difference in the mean defibrillation energy per kilogram between the two configurations $(1.17 \pm 0.63$ joules $/ \mathrm{kg}$ for configuration $\mathrm{B}$ and $1.06 \pm 0.23$ joules $/ \mathrm{kg}$ for configuration $\mathrm{A}$; $\mathrm{P}=\mathrm{NS})$.

Mean weight was $24.4 \pm 2.8 \mathrm{~kg}$ in group 2 $(\mathrm{P}=0.010$ vs group 1$)$. One hundred and fourteen of 214 shocks were successful at defibrillation (53\%). Forty-three shocks (40\%) were successful using configuration A (RV apex to common SVC distal CS) and $71(66 \%)$ were successful using configuration C (RV apex to superior vena cava). This difference was significant at the $\mathrm{P}<0.001$ level. Mean defibrillation energy at which $50 \%$ or more of shocks were successful (Fig. 3) was significantly lower using configuration $\mathrm{C}(21.4 \pm 9.0)$ than configuration $\mathrm{A}(27.1 \pm 9.5 ; \mathrm{P}<0.01)$. Mean $\mathrm{E}_{50}$ was $0.89 \pm$ 0.4 joules $/ \mathrm{kg}$ for configuration $\mathrm{C}$ and $1.12 \pm 3.8$ joules $/ \mathrm{kg}(\mathrm{P}<0.01)$ for configuration $\mathrm{A}$.

\section{Voltage Measurements}

Shock amplitude and duration were measured during test pulses using each of the configurations. Mean leading edge voltage was similar in the three configurations $(622 \pm 137$ volts for configuration $A, 610 \pm 131$ volts for configuration $\mathrm{B}$, and $617 \pm 151$ volts for configuration C). However, pulse duration was significantly shorter when using a catheter configuration including the coronary sinus catheter (configurations B and C). Mean pulse duration was $11.3 \pm 2.8 \mathrm{msec}$ for configuration $\mathrm{A}, 6.6 \pm$ $1.8 \mathrm{msec}$ for configuration $\mathrm{B}$, and $6.1 \pm 1.5 \mathrm{msec}$ for configuration $C(P<0.01$ configurations $B$ and $C$ vs configuration $A$ ). To determine the effects of catheter location within the coronary sinus, voltage measurements were made with a catheter tip in the proximal coronary sinus and compared to those with a catheter tip in the posterior coronary sinus. Mean pulse duration was $10.5 \pm 2 \mathrm{msec}$ in the proximal coronary sinus and $6.0 \pm 2.2 \mathrm{msec}$ in the lateral coronary sinus $(\mathrm{P}<0.05)$.

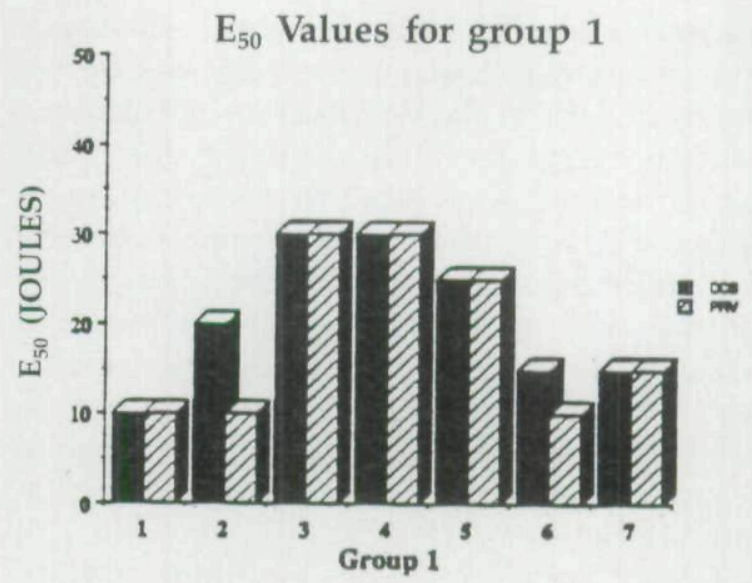

Figure 2. Energy in joules at which $50 \%$ or more of shocks were successful for the animals in group 1. Values were the same in four of the six animals and higher for the right ventricular apex to coronary sinus configuration in two of the animals. There was no difference in the mean energy at which $50 \%$ or more of the shocks were successful using the two configurations.

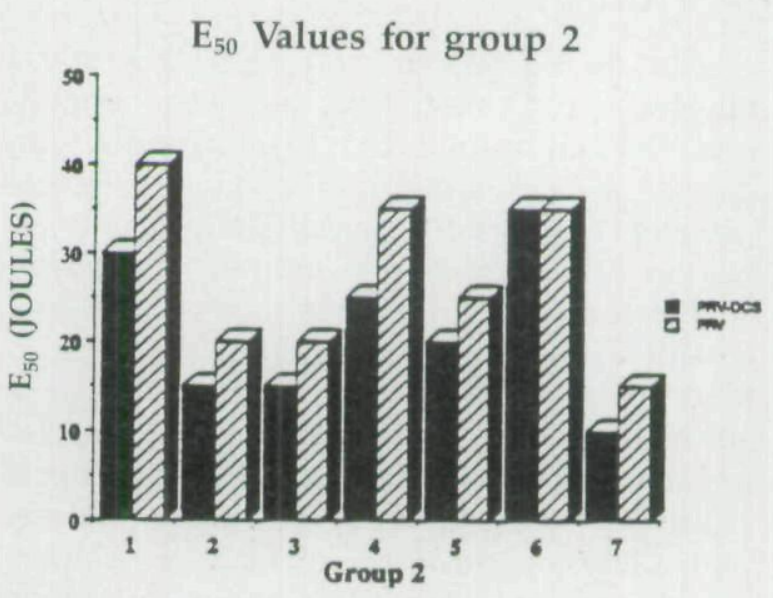

Figure 3. Energy in joules at which $50 \%$ or more of shocks were successful for the animals in group 2. Values were lower for the three-electrode configuration in six animals of the seven animals and similar in one. This difference was significant at the $P<0.01$ level.

\section{Adverse Effects}

Despite the delivery of up to 38 shocks (including rescue shocks) through the coronary sinus catheter, no instances of hemorrhage, per- 
foration or visible damage to the coronary sinus occurred. Data on arrhythmias was not available for two group 1 animals.

Nonsustained episodes of ventricular tachycardia and transient complete heart block were seen frequently. A total of 162 episodes of nonsustained ventricular tachycardia with a mean rate of $211 \pm 31$ beats/min and mean duration of $15 \pm 10$ seconds were seen. The occurrence of ventricular tachycardia did not appear to be related to energy level but because of the study design, only a narrow range of energy levels (near the defibrillation threshold) were frequently tested in each animal. In group 1 , nonsustained ventricular tachycardia occurred in 40 of $68(59 \%)$ defibrillation attempts using configuration B and 14 of 68 (21\%) attempts using configuration $\mathrm{A}(\mathrm{P}<0.001)$. In group 2 , nonsustained ventricular tachycardia was equally frequent in configuration $\mathrm{A}$ (50 of $107 ; 48 \%$ ) and configuration C (58 of $107 ; 54 \%$ ).

Transient complete heart block was observed frequently, but the duration could not be precisely determined because back-up pacing was begun at a rate of 117 beats/min for asystole. Except in one group 1 animal, heart block was always resolved by the time of delivery of the next shock. The incidence of heart block was not different between the two configurations in either group. In group 1, 11 of 53 shocks (21\%) delivered between the distal CS and right ventricular (RV) apex (configuration A) were associated with complete heart block versus 6 of $53(11 \%)$ with configuration $B(P=N S)$. Four of five of the animals in which arrhythmia data was available developed at least one episode of complete heart block. In group 2, 22 of 107 (21\%) shocks delivered with configuration A developed complete heart block versus 15 of 197 with configuration $\mathrm{C}(\mathrm{P}=\mathrm{NS})$. All but one of seven animals developed at least one episode of transient complete heart block.

\section{Discussion}

The major finding of this study is that an electrode configuration including a transvenous catheter in the coronary sinus reduces defibrillation thresholds in comparison to a bipolar superior vena cava to right ventricular apex configuration. Although bipolar defibrillation from the coronary sinus to right ventricular apex did not significantly reduce defibrillation threshold, the use of the superior vena cava and coronary sinus as a common cathode did. Despite up to 38 shocks in individual animals, no damage to the coronary sinus was seen. Thus, the use of a catheter in the coronary sinus may prove practical in order to reduce transvenous defibrillation thresholds.

\section{Defibrillation Thresholds}

Successful defibrillation depends on the delivery energy and electrode location. Several studies have addressed the energy dependence of defibrillation. Defibrillation is a statistical phenomenon without a single clear cutoff energy between successful and unsuccessful defibrillation. Gold et al. ${ }^{11}$ suggested in 1979 that a single cutoff energy was not appropriate to characterize defibrillation efficacy and devised a contour graph relating percent success of defibrillation to the energy characteristics of the device. More recently, Davey et al. ${ }^{12}$ showed that in dogs subjected to internal defibrillation there was no clear cutoff energy between successful and unsuccessful energies. They constructed dose response curves for defibrillation versus energy, and suggested analyzing defibrillation success in individual animals to improve the sensitivity in detecting differences between defibrillation methods. Although other investigators have supported the concept of a defibrillation threshold, data in the current study was analyzed using the level of energy at which at least $50 \%$ of shocks of $E_{50}$ produced successful defibrillation. A total of up to five shocks were delivered at each energy level using each configuration.

\section{Determinants of Defibrillation}

Differing shock waveforms, energy levels, electrode positions, body and heart weight, cardioactive drugs and ischemia have been shown to affect energy requirements for defibrillation. ${ }^{13-16}$ The location of defibrillating elec- 
trodes also has a major effect on defibrillation efficacy. Although it had previously been hypothesized that a specific location of defibrillation electrodes was necessary for adequately depolarizing a critical mass of myocardium, it has recently been shown that even unsuccessful shocks can lead to depolarization over the entire heart. ${ }^{9,17}$ However, the isoelectric interval before resumption of fibrillation was shorter in unsuccessful shocks and ventricular fibrillation regenerated at sites distal from the defibrillating electrodes. Thus, although simple depolarization of distal myocardial sites is not adequate to insure successful defibrillation, the current density around such sites and thus defibrillating electrode location, can affect defibrillation efficacy. In the current study, catheters were positioned in the lateral coronary sinus and right ventricle in an attempt to interpose large amounts of myocardium between the electrodes.

\section{Defibrillation Lead Systems}

Clinical defibrillators use two large patches placed directly on the epicardial surface of the heart or a high right atrial superior vena cava lead combined with an epicardial patch. ${ }^{7}$ This approach has resulted in defibrillation thresholds that have generally been $<30$ joules, but requires epicardial implantation of at least one lead. Experimental approaches to other lead systems have included the use of a subcutaneous patch to right-sided electrodes in an attempt to reduce current pathways across large portions of the left ventricle during defibrillating shocks efficacy. ${ }^{18}$ Recent clinical trials have demonstrated some success with this approach. 8,9 Several studies have examined three-electrode configurations. ${ }^{15,19-22}$ These have demonstrated a significant reduction in the requirement of defibrillation energy. A recent study has shown that it is electrode separation rather than the sequential nature of the shocks that reduces defibrillation thresholds. ${ }^{20}$ Specifically, there was no difference between defibrillation thresholds using the three-electrode system with simultaneous versus sequential shocks.
Two separate lead systems were evaluated in the current study. A catheter in the distal coronary sinus had no greater defibrillating efficacy than one in the SVC-right atrium when combined with one right ventricular apex. However, the RVA-CS shocks were associated with a shorter pulse duration suggesting a low resistance pathway was present. This may have been related to catheter design. The proximal edge of the distal electrode was located $4.5 \mathrm{~cm}$ from the catheter tip. Thus, the right ventricular and coronary sinus electrodes both may have been in part located in the right atrium and thus allowed current shunting. This hypothesis is supported by the fact that defibrillating pulse duration was longer when in large animals the catheter was placed very distally in the coronary sinus. Using a catheter with shorter electrodes may eliminate this difficulty. ${ }^{8,9}$

Several studies have examined three-pole configurations for defibrillation. Many of these have used sequential shocks using two separate anode cathode configurations have previously examined a coronary sinus electrode as part of a sequential shock system, but they did not systematically test the effect of this electrode on defibrillation thresholds.

A three-electrode configuration was also evaluated in this study. Although pulse width was also approximately $6 \mathrm{msec}$ in duration (suggesting some current shunting), energy requirements for defibrillation were lower than control using a common high right atrial coronary sinus cathode. Thus, this configuration could potentially contribute to an effective transvenous defibrillating system.

\section{Limitations}

There are several limitations to the current study. Defibrillation was tested in an openchested model and current pathways could potentially be different in such a situation. However, this should be less of a problem in testing a transvenous system than an epicardial one and a recent study showed that even with epicardial patches there was no difference in defibrillation threshold between open- and 
closed-chested animals. ${ }^{24}$ In addition, testing was performed on mongrel dogs without heart disease and there may be limitations in applying these findings to patients with organic heart disease.

Only a single polarity for the right ventricular apex electrode (positive) was evaluated in this study. Official recommendations have suggested that the left ventricular patch electrode always has negative polarity. However, a recent study by Bardy et al. ${ }^{25}$ showed that defibrillation thresholds were actually lower when the left ventricular patch was negative. It is possible that if the coronary sinus electrode had served as the anode, even lower defibrillation thresholds would have been found since this catheter was in closest proximity to the left ventricle. However, the fairest comparison to the control (RV apex to SVC) configuration involved using the right ventricular apex as the anode since it was the only electrode in proximity to the left ventricular myocardium in both configurations.

The absolute value of defibrillation threshold for the control configuration was substantially higher in group 2 than in group 1. However, weight which may be a determinant of defibrillation threshold was higher in group 2 and the mean defibrillation threshold per $\mathrm{kg}$ was similar in the two groups.

No gross damage to the coronary sinus was observed in this study. However, histologic analysis after chronic lead implantation was not performed and will be required in future studies before the technique can be deemed safe.

\section{Clinical Implications}

The results of this study suggest that a catheter in the coronary sinus could potentially form a part of an effective transvenous defibrillating system. Although the mean defibrillation energy using this configuration was still relatively high, the use of bipolar waveforms and improvements in catheter design including shortening the electrodes could potentially reduce energy requirements to the point where they could be clinically useful. Further work including chronic studies on damage to the coronary sinus and incorporating changes in catheter design and waveform pulses will be necessary to confirm the feasibility of this approach.

Acknowledgments: We would like to thank Lisa Stephens for her assistance in preparing the manuscript. We would also like to thank CPI, Inc. (St. Paul, MN, USA) for providing the external cardioverter defibrillator and catheters for use in this study.

\section{References}

1. Mirowski M, Morton MM, Langer A, et al: A chronically implanted system for automatic defibrillation in active conscious dogs. Circulation 58:90, 1978 .

2. Mirowski M, Reid PR, Watkins L, et al: Clinical treatment of life-threatening ventricular tachyarrhythmias with the automatic implantable defibrillator. Am Heart J 102:265, 1981.

3. Echt DS, Armstrong K, Schmidt P, et al: Clinical experience, complications and survival in $70 \mathrm{pa}-$ tients with the automatic implantable cardioverter/defibrillator. Circulation 71:289, 1985.

4. Fogoros RN, Fiedler SB, Elson JJ: The automatic implantable cardioverter-defibrillator in drugrefractory ventricular tachyarrhythmias. Am Coll Phys 107:635, 1987.

5. Mirowski M, Reid PR, Winkle RA, et al: Mortality in patients with implanted automatic defibrillators. Ann Intern Med 98:585, 1983.

6. Deeb GM, Griffith BP, Thompson ME, et al: Lead systems for internal ventricular fibrillation. Circulation 61:242, 1981.

7. Troup PJ: Lead system selection, implantation, and testing for the automatic implantable cardioverter-defibrillator. Clin Prog Electrophysiol Pacing 4:260, 1986.

8. Saksena S, Parsonnet V: Implantation of a cardioverter/ defibrillator without thoracotomy using a triple electrode system. J Am Coll Cardiol 259:69, 1988.

9. Winkle RA, Bach SM Jr, Mead RH, et al: Comparison of defibrillation efficacy in humans using a new catheter and superior vena cava spring-left ventricular patch electrodes. J Am Coll Cardiol 11:365, 1988.

10. Chen PS, Shibata N, Dixon EG, et al: Activation during ventricular defibrillation in open-chest dogs. J Clin Invest 77:810, 1986.

11. Gold JH, Schuder JC, Stoeckle H: Contour graft for relating percent success in achieving ventricular defibrillation to duration, current, and energy content of shock. Am Heart J 98:207, 1979. 
12. Davey J, Fain ES, Dorian P, et al: The relationship between successful defibrillation and delivered energy in open-chest dogs: Reappraisal of the defibrillation threshold concept. Am Heart J 113:77, 1987.

13. Chapman PD, Vetter JW, Souza JJ, et al: Comparative efficacy of monophasic and biphasic truncated exponential shocks for nonthoracotomy internal defibrillation in dogs. J Am Coll Cardiol 12:739, 1988.

14. Rubin L, Hudson P: Epicardial versus parietal pericardial defibrillation. Am J Emerg Med 3:160, 1985.

15. Jones DL, Klein GJ, Rattes MF, et al: Internal cardiac defibrillation: Single and sequential pulses and a variety of lead orientations. PACE 11:583, 1988.

16. Kerber RE, Pandian NG, Hoyt R, et al: Effect of ischemia, hypertrophy, hypoxia, acidosis, and alkalosis on canine defibrillation. Am J Physiol 244:H825, 1983.

17. Chen $\mathrm{P}$, Wolf $\mathrm{PD}$, Claydon FJ, et al: The potential gradient field created by epicardial defibrillation electrodes in dogs. Circulation 74:626, 1986.

18. Wetherbee JN, Chapman PD, Klopfenstein HS, et al: Nonthoracotomy internal defibrillation in dogs: Threshold reduction using a subcutaneous chest wall electrode with a transvenous catheter elec- trode. J Am Coll Cardiol 10:406, 1987.

19. Moore TW, DiMeo FN, Dubin SE: The effect of shock separation time on multiple-shock defibrillation. Med Instrum 12:31, 1978.

20. Wetherbee JN, Chapman PD, Bach SM Jr, et al: Sequential shocks are comparable to single shocks employing two current pathways for internal defibrillation in dogs. PACE 11:696, 1988.

21. Hannekum A, Dalichau H, Kochs M, et al: Sequential or single pulse defibrillation? Investigation towards energy reduction in experimental animals. Thorac Cardiovasc Surg 35:270, 1987.

22. Jones DL, Klein GJ, Kallok MJ: Improved internal defibrillation with twin pulse sequential energy delivered to different lead orientations in pigs. Am J Cardiol 55:821, 1985.

23. Bardy $G H$, Stewart RB, Ivey $T D$, et al: Intraoperative comparison of sequential-pulse and single-peulse defibrillation in candidates for automatic implantable defibrillators. Am J Cardiol 60:618, 1987.

24. Kallok MJ: The influence of opening the thorax on defibrillation threshold in canines. PACE 12:70, 1989.

25. Bardy GH, Ivey TD, Allen MD, et al: Evaluation of electrode polarity on defibrillation efficacy. Am J Cardiol 63:433, 1989. 
This document is a scanned copy of a printed document. No warranty is given about the accuracy of the copy. Users should refer to the original published version of the material. 